\title{
The evaluation of clinical outcomes of a pre-bariatric surgery medical
}

\section{Countess of Chester Hospital N/FS}

\author{
NHS Foundation Trust
}

hlachakr@liverpool.ac.uk; sunilnair1@nhs.net

1. Institute of Ageing \& Chronic Disease, University of Liverpool. 2. Countess of Chester Hospital NHS Foundation Trust.

\section{Introduction}

Obesity is a public health challenge. In the UK, $24 \%$ of adults are obese ${ }^{1}$. Obesity may lead to complications including type 2 diabetes mellitus, ischaemic heart disease (IHD), obstructive sleep apnoea syndrome (OSAS), and certain psychological disorders ${ }^{2}$

A tiered, weight management service model was designed by the National Institute for Health and Care Excellence (Figure 1), to aid lifestyle changes such as modifications in diet, exercise, and behaviour in patients who are obese $\mathrm{e}^{3}$ Tier-3 services were designed to facilitate such changes, with appropriate multidisciplinary specialist input. Currently, there is little published evidence of the evaluation of tier-3 services ${ }^{4}$.

The STOP-BANG questionnaire is a screening tool for OSAS, consisting of eight dichotomous items. The likelihood of a patient suffering from moderatesevere OSAS, with a STOP BANG score of $\geq 3$ and $\geq 5$, is $25 \%$ and $45 \%$, respectively. There is much dispute about what the cut-off score should be, to diagnose OSAS

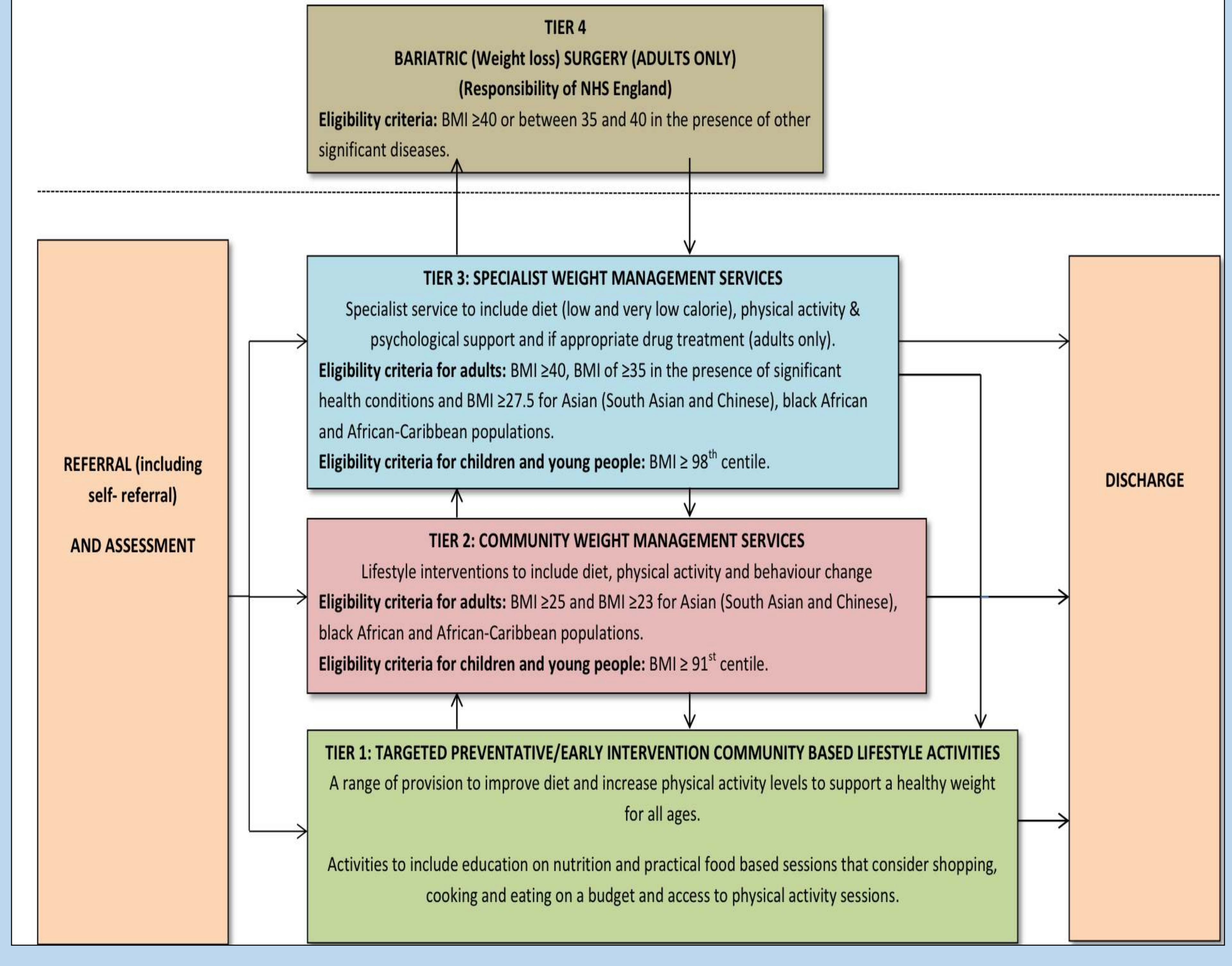
$\begin{aligned} & \text { Figure } 1 \mid \text { 4-tier weight management service model outlining the bariatric pathway, which has been designed by the National } \\ & \text { Institute of Health and Care Excellence. N.B. This figure is taken from a study published by Nottinghamshire County Council'. }\end{aligned}$

\section{Aims}

- To investigate clinical outcomes (as measures of physical and mental health) of patients who are obese, and use the tier-3 services at the Countess of Chester Hospital ( $\mathrm{COCH})$

To evaluate the effectiveness of using the STOP-BANG questionnaire for diagnosing OSAS.

\section{Methodology}

This cohort study included 234 patients who used tier-3 services at $\mathrm{COCH}$, between April 2013 and January 2017. The focus was on analysing data that was previously collected for routine patient care. Patients' BMI, weight, total cholesterol (a risk factor for IHD), and $\mathrm{HbA1c}$ (a measure of glucose control) were compared, before and after tier-3 service usage. Patients' STOP BANG scores and whether they received continuous positive airway pressure (CPAP) therapy (a treatment for OSAS) were analysed.

At $\mathrm{COCH}$, tier-3 service users are offered cognitive

behavioural therapy (CBT), if necessary. The patient scores of three psychometric measures were compared, before and after CBT. The patient health questionnaire (PHQ9) and CORE-10 measures test for depression severity and any symptoms. The weight efficacy lifestyle (WEL) questionnaire assesses how well patients resist unhealthy eating.

\section{Results}

Clinical Outcomes: Physical Health

There were significant reductions in mean BMI, HbA1c and total cholesterol (Wilcoxon signed-rank tests; $p=0.001$ ) by $3.66 \%, 9.05 \%$ and $8.75 \%$, respectively, following the use of tier-3 services. Similarly, there was a significant reduction in weight, by $3.58 \%$ (paired samples $t$-test; $\mathrm{p}=0.001, \mathrm{t}=6.070, \mathrm{df}=151$ ) (Figure 2).

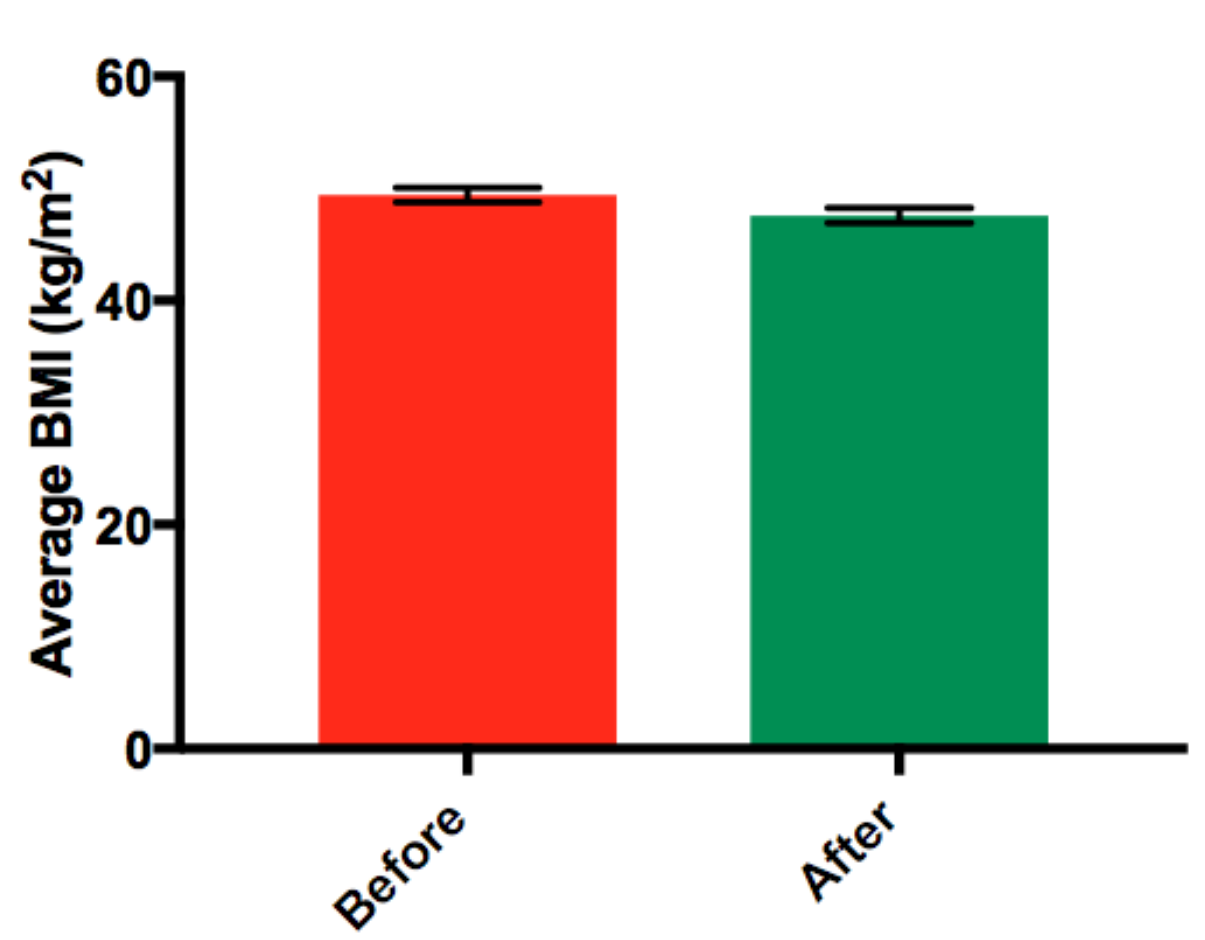

B)

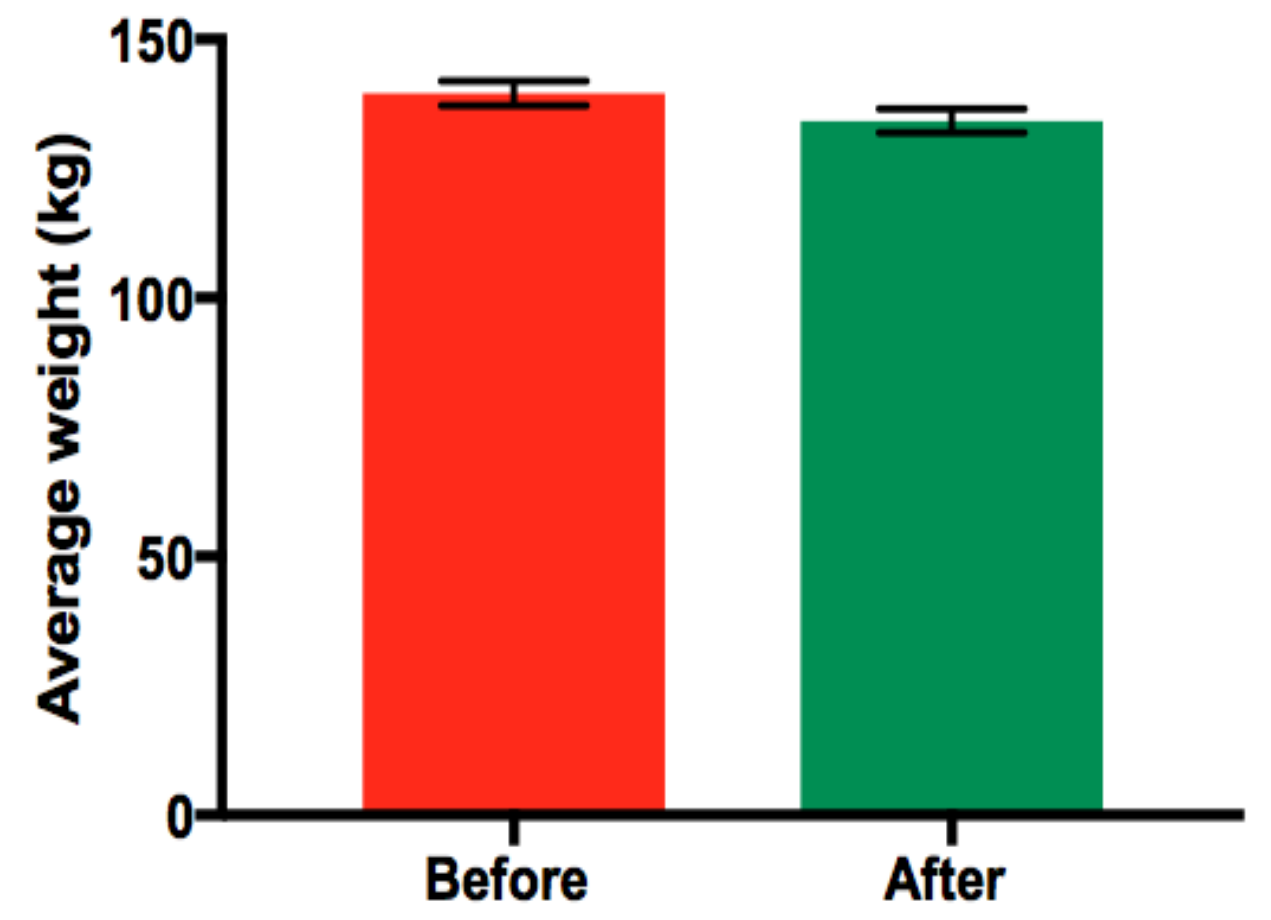

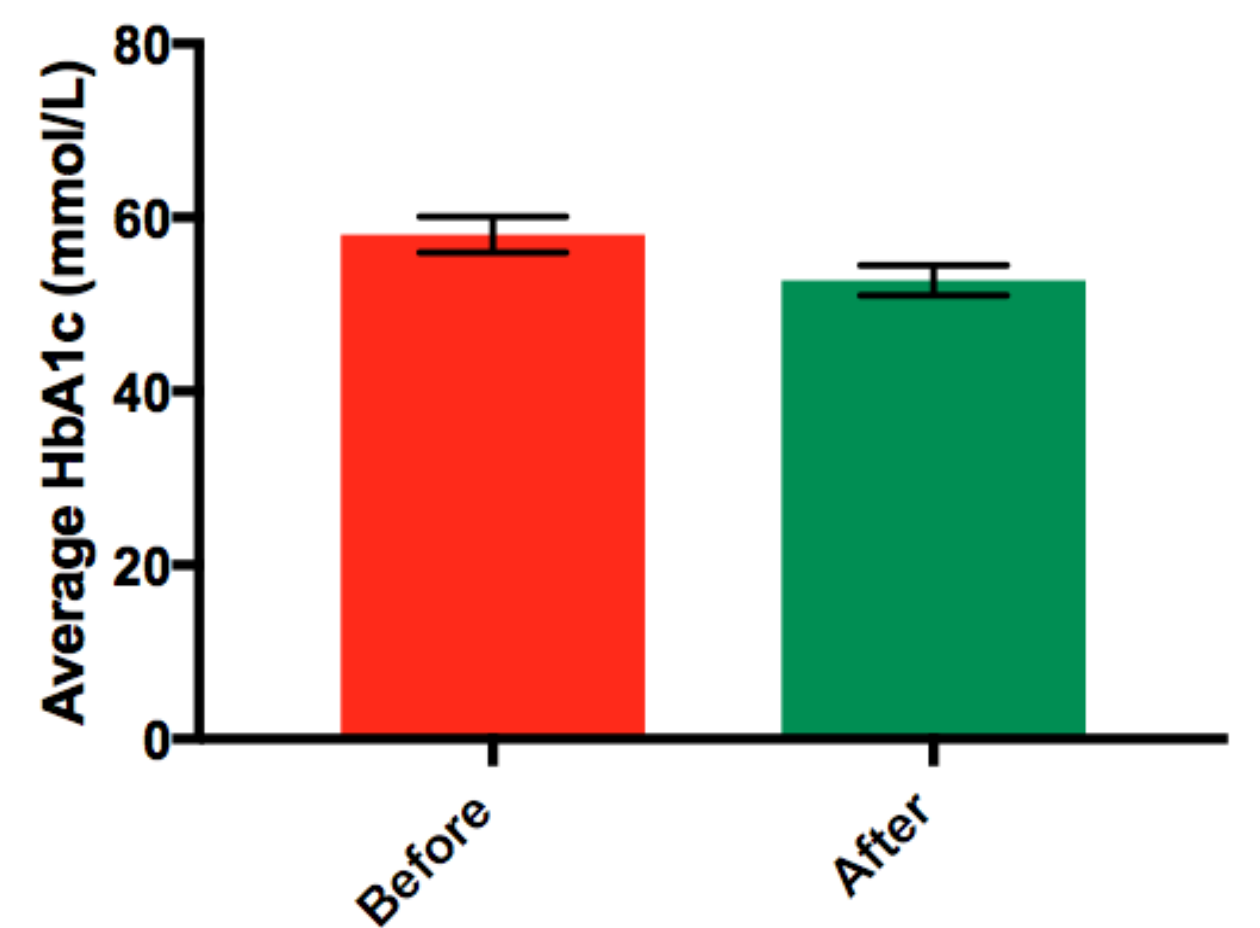

D)

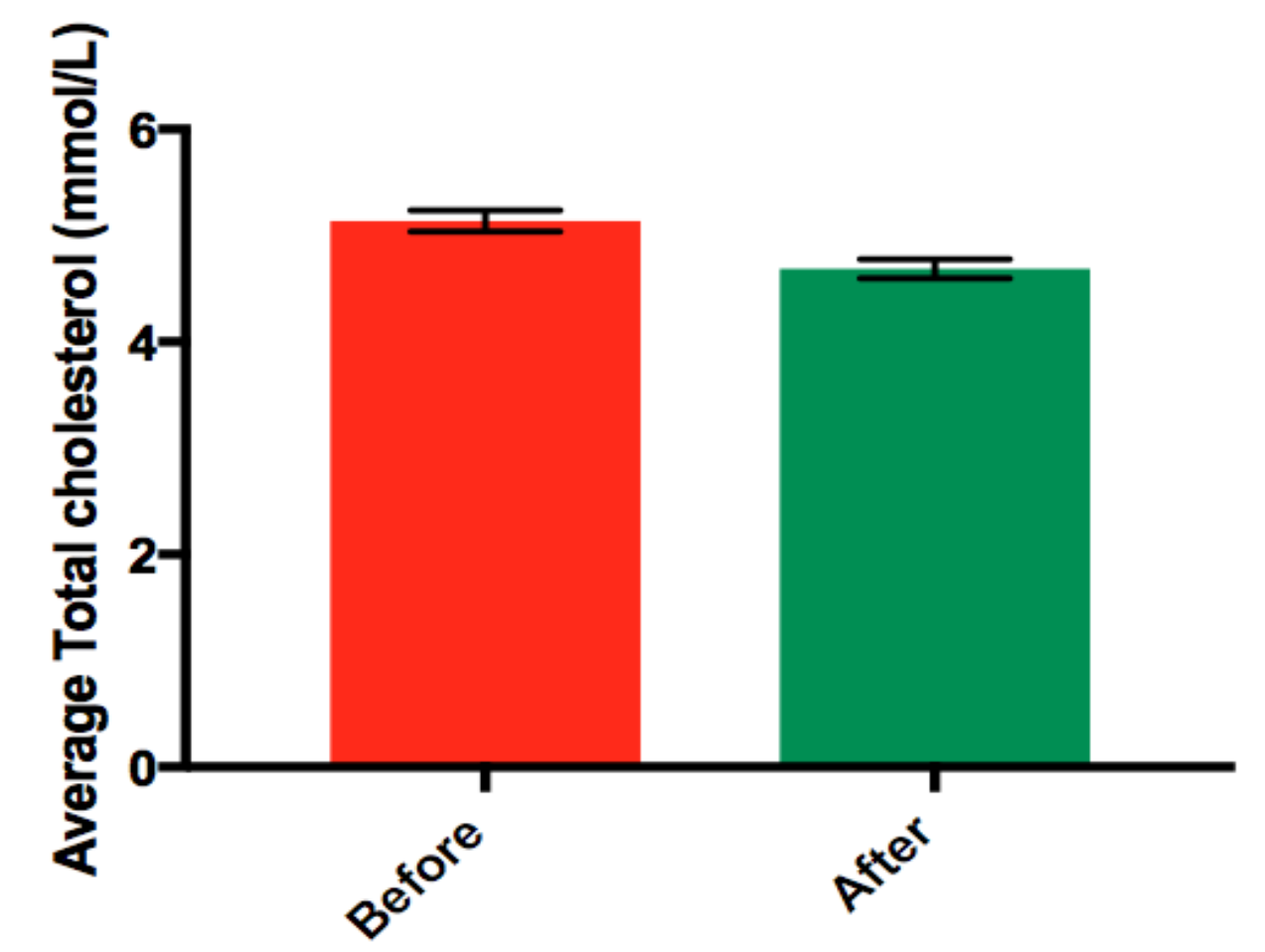

Figure 2 | Changes to BMI, Weight, HbA1c, and Total Cholesterol: A) Mean BMI, B) Mean weight, C) Mean HbA1c, and D) Mean total Chole

\section{STOP BANG questionnaire and corresponding CPAP therapy}

Out of 84 patients that had a STOP BANG score $\geq 3,47.62 \%$ of them were administered CPAP therapy. The proportion of patients with a STOP BANG score between 3 and 5 , and those with a score $>5$, that received CPAP therapy, were 31.25 and $55.1 \%$, respectively (Figure 3).
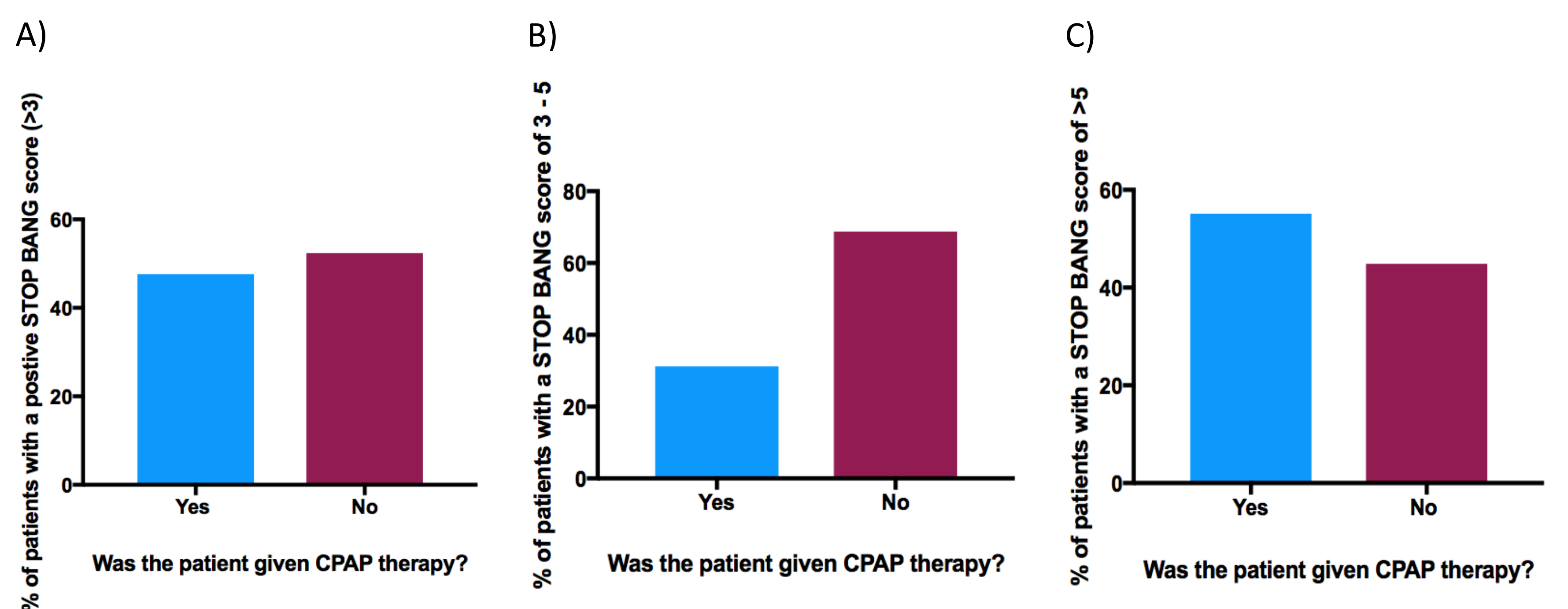

Figure 3 | STOP BANG scores and its association with patients receiving CPAP therapy: A) Positive STOP BANG (>3), B) STOP BANG score between 3 to 5 , and C) STOP BANG score $>5$.
Psychometric measures

There were significant improvements (Wilcoxon signed-rank test; $p=0.001$ ) in the psychometric measures, with a decrease of 7.8 and 6.71 , for the PHQ-9 and CORE-10 scores, respectively, and an increase in the WEL score by 2.29, following CBT (Figure 4).

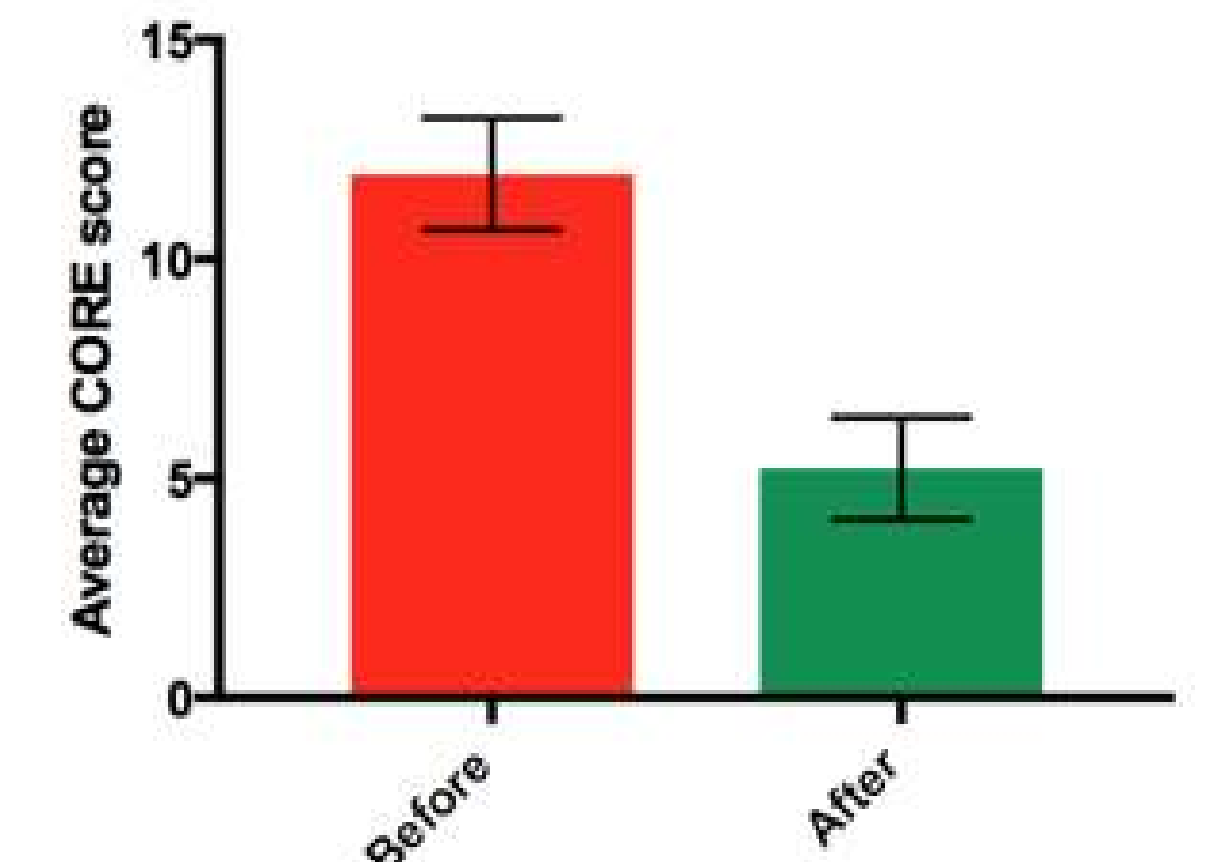

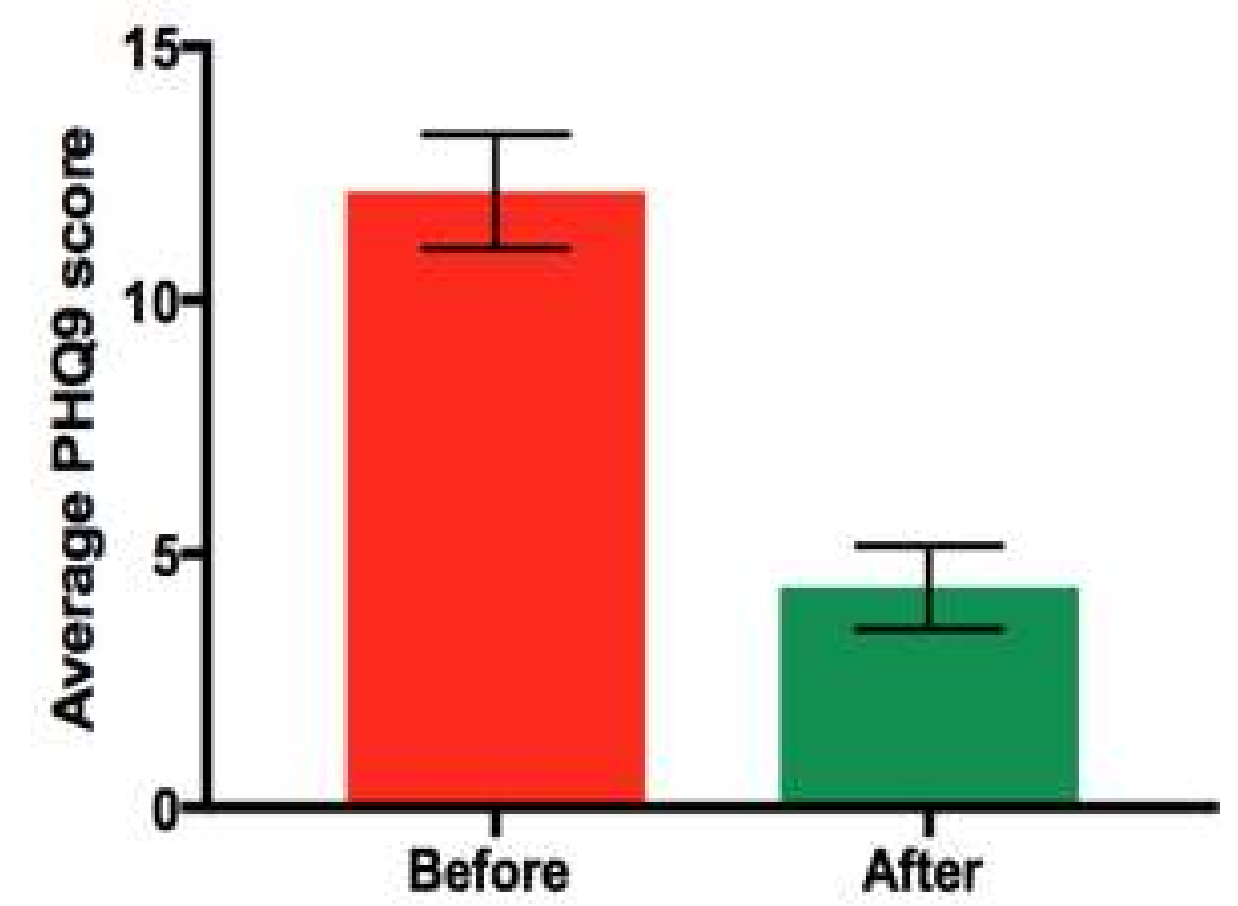

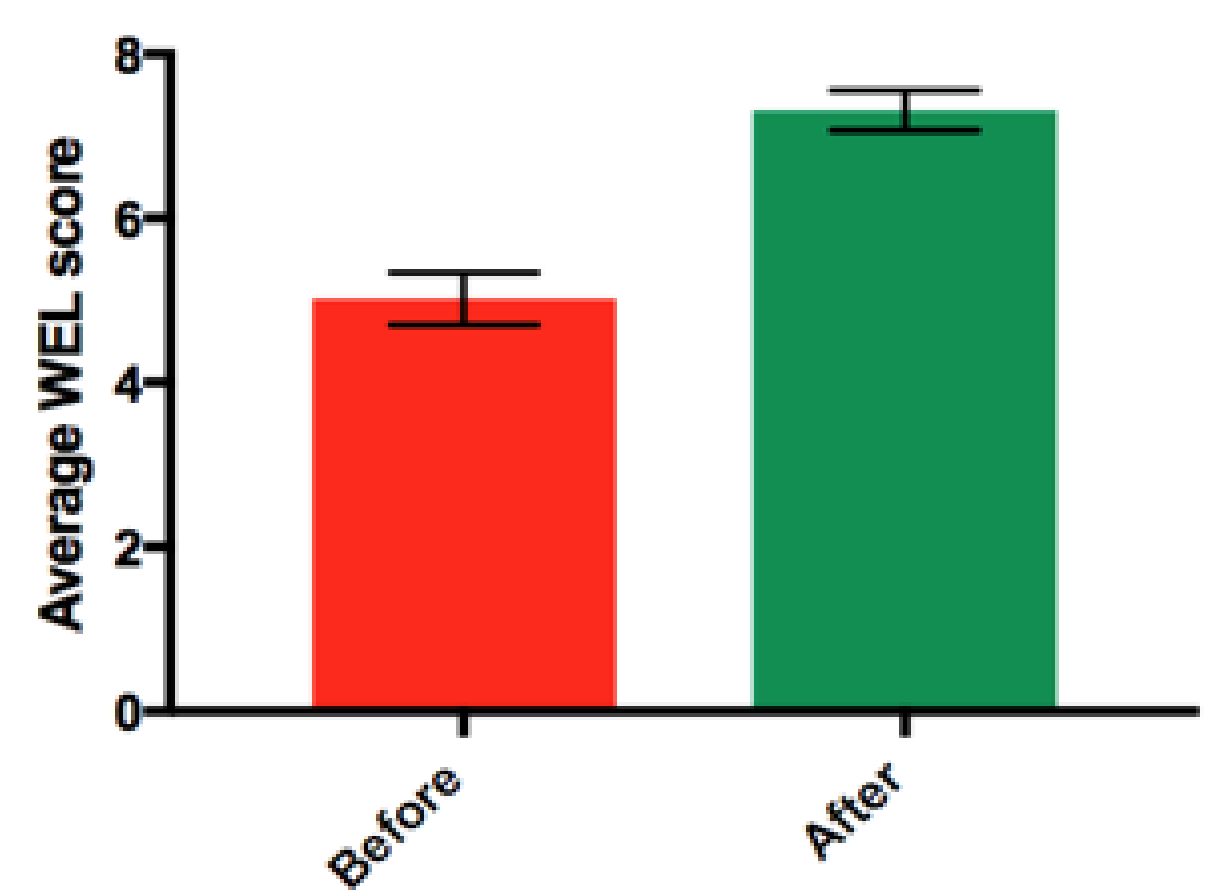

Figure $4 \mid$ Changes to PHQ-9, CORE-10 and WEL scores: $A) \mathrm{T}$ and after the use of CBT ( \pm Standard Error of the Mean).

\section{Conclusions}

This study suggests that the STOP BANG questionnaire is suitable for screening for OSAS. However, the small sample size ( $n=16)$ for the cohort of patients with a score between 3 and 5 suggests that there is insufficient evidence to conclude what the ideal cut off score should be, to be considered a positive test.

In comparison to improvements in the clinical outcomes concerning physical health, the tier-3 services at COCH have shown an improvement in the mental health of the service users to a greater extent. This study emphasises that comprehensive medica interventions, especially those that focus on lifestyle changes can significantly reduce the BMI and the likelihood of complications associated with obesity occurring, thus highlighting its importance prior to referral for bariatric surgery.

\begin{tabular}{|l|}
\hline References \\
\hline 1. House of Commons, 2014. Obesity Statistics. [pdf] House of \\
Commons Library. \\
2. Segula D, 2014. Malawi medical journal, 26(1):20-4. \\
3. National Institute for Health and Care Excellence, 2014. \\
Weight management: lifestyle services for overweight or obese \\
adults. [pdff National Institute for Health and Care Excellence. \\
4. Nottinghamshire County Council, 2013. Obesity Prevention \\
and Weight Management Consultation Summary. [pdf] \\
Nottinghamshire County Council. \\
5. Nagappa A, et al., 2015. Public Library of Science One, \\
10 (12):e0143697. \\
\hline
\end{tabular}

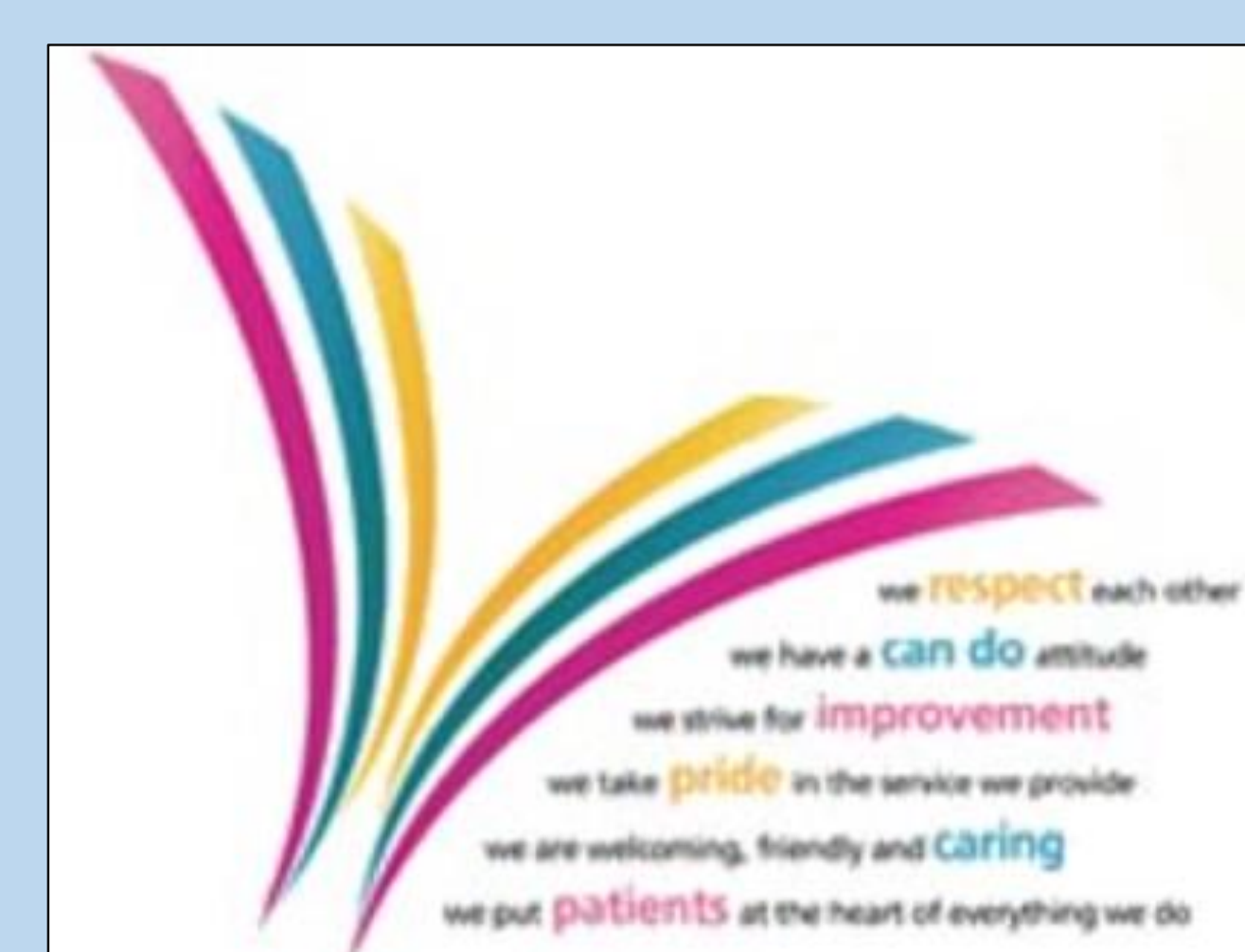

